Document downloaded from:

http://hdl.handle.net/10251/157277

This paper must be cited as:

Zheng, D.; Cai, Z.; Floris, I.; Madrigal-Madrigal, J.; Pan, W.; Zou, X.; Sales Maicas, S. (2019). Temperature-insensitive optical tilt sensor based on a single eccentric-core fiber Bragg grating. Optics Letters. 44(22):5570-5573. https://doi.org/10.1364/OL.44.005570

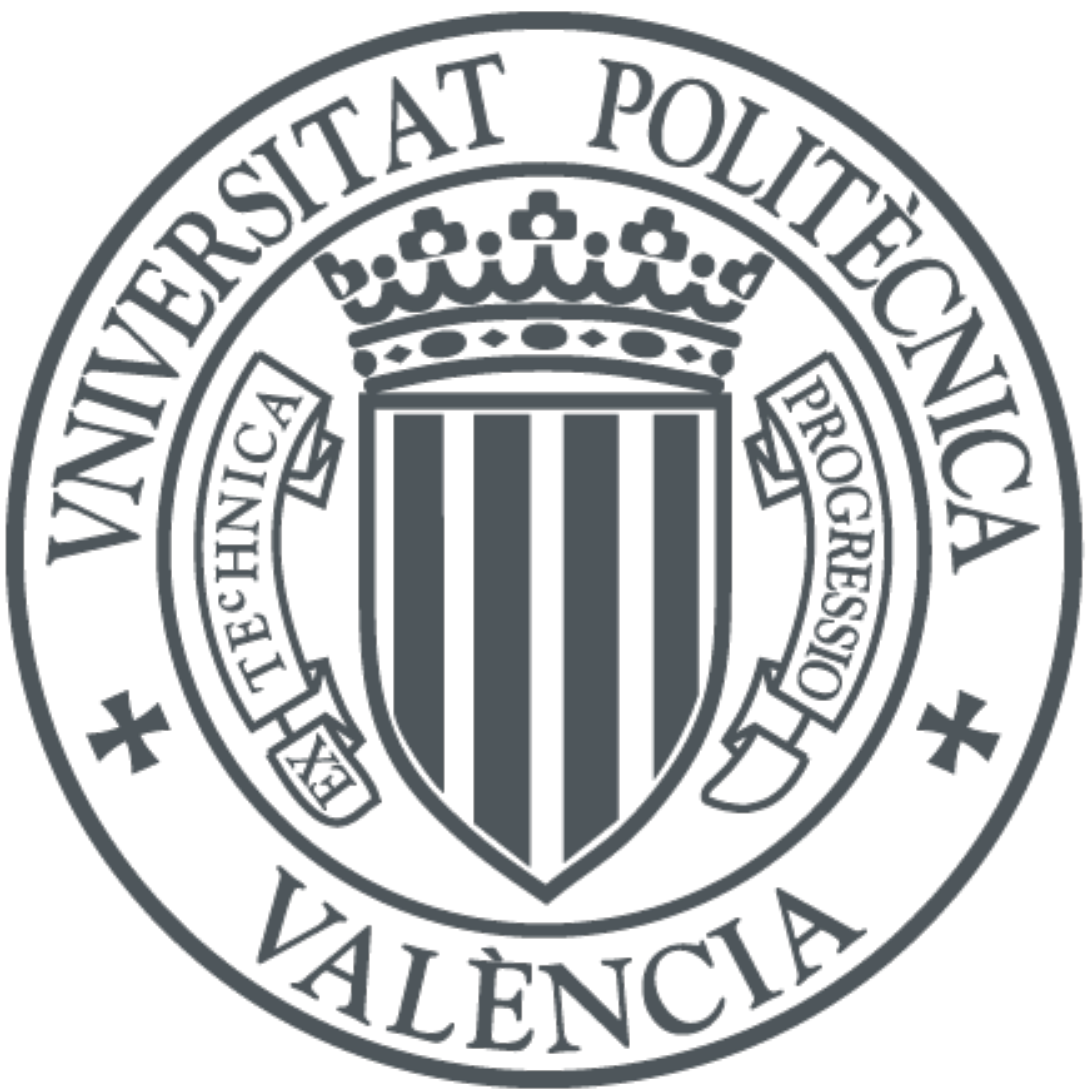

The final publication is available at

https://doi.org/10.1364/OL.44.005570

Copyright The Optical Society

Additional Information 


\title{
Temperature-insensitive optical tilt sensor based on a single eccentric-core fiber Bragg grating
}

\author{
Di Zheng, ${ }^{1,2 *}$ Zhuyang Cal, $^{1}$ Ignazio Floris, ${ }^{2}$ JAVIer Madrigal, ${ }^{2}$ Wei Pan, ${ }^{1}$ \\ Xihua Zou, ${ }^{1}$ and Salvador Sales ${ }^{2}$ \\ ${ }^{1}$ Center for Information Photonics \& Communications, School of Information Science \& Technology, Southwest Jiaotong University, Chengdu, \\ 610031, China \\ 2 Photonics Research Labs, ITEAM Research Institute, Universitat Politècnica de València, Camino de Vera, s/n, 46022, Valencia, Spain \\ *Corresponding author: $\underline{d z h e n g @ h o m e . s w j t u . e d u . c n}$
}

Received XX Month XXXX; revised XX Month, XXXX; accepted XX Month XXXX; posted XX Month XXXX (Doc. ID XXXXX); published XX Month XXXX

\begin{abstract}
This paper presents a novel and simple temperatureinsensitive optical tilt sensor based on a single eccentriccore fiber Bragg grating (ECFBG). By partly inserting an ECFBG into a ceramic ferrule, the reflection spectrum of the ECFBG splits into two peaks as a result of the applied tilt angle. The magnitude and direction of inclination in one-dimension can be determined by monitoring the wavelength separation between both peaks, which is inherently insensitive to temperature. The proposed tilt sensor has a good linear response within a wide dynamic range of $\pm 45^{\circ}$, with a sensitivity of $0.012 \mathrm{~nm} /{ }^{\circ}$, resolution of $0.83^{\circ}$ and accuracy of $0.41^{\circ}$. Being free from any inherent mechanical joint/ friction, along with the advantages of a compact structure, good repeatability and low cost, the proposed sensor is highly suitable for practical engineering applications.
\end{abstract}

OCIS codes: (060.3735) Fiber Bragg gratings; (060.2370) Fiber optics sensors.

http://dx.doi.org/10.1364/OL.99.099999

Tilt sensors, or inclinometers, are widely used in fields such as civil engineering, mechanical manufacturing, robotics and aeronautical engineering [1,2]. So far, various types of tilt sensors have been developed based on different operating principles, such as resistive [3], capacitive [4] inductive [5] and fiber-optic effects $[6,7]$. Compared with other types of tilt sensors, fiber-optic based tilt sensors exhibit merits in terms of their immunity to electromagnetic interference, remote sensing capability, intrinsic safety, high sensitivity and compact size [8,9]. Particularly, fiber Bragg gratings (FBG) based tilt sensors have attracted considerable research interest due to their inherently selfreferencing nature and the multiplexing capability.

Over the past two decades, a lot of research efforts have been devoted to developing high-performance FBG based tilt sensors. The majority of the previously reported design strategies were based on a pendulum structure and suspension configuration [1016]. Although these two solutions have been shown to be an effective way of improving sensor performance in terms of sensitivity, accuracy and resolution, there are still several inherent shortcomings existing in current schemes, that make them impractical for actual sensing applications. Firstly, pendulumbased inclinometers generally suffer from undesirable mechanical friction, rotation, and a complicated strain transfer between the pendulum structure and the FBG, which makes it difficult to obtain high accurate measurements and good repeatability. In the case of suspension-structure-based tilt sensors, which are free from inherent mechanical joints/friction, the FBGs are stretched directly without any protection so that there is a high risk of damage. In addition, these two types of device are usually bulky and complicated, which means it is hard and costly to embed them in the structure. Secondly, since the FBG is inherently sensitive to both strain and temperature, to make the tilt-sensor temperatureinsensitive, it is necessary to place two FBGs as close together as possible to compensate for the temperature induced wavelength shift, or to place the sensor in an isothermal environment to avoid temperature variations. Although MacPherson et al. have reported the use of a multicore fiber incorporating several FBGs to minimize temperature sensitivity by enclosing multiple cores in the same cladding, a specifically designed fan-out device is necessary to extract strain information from different cores [17]. Thirdly, as one-dimensional tilt sensing is dependent on the differential strain measured between two FBGs, two independent interrogation operations are typically required, which makes the interrogation procedure time-consuming.

To address the above- mentioned limitations, we designed and tested a simple and innovative tilt sensor with the ability to measure the magnitude and direction of inclination. The proposed sensor was produced by inscribing a single FBG in eccentric-core fiber (ECF), which enables easy fabrication and interrogation operations. By partially fixing the ECFBG, the ECFBG spectrum splits into two different resonant peaks when the tilt angle is applied. The wavelength separation between the two peaks is 
proportional to the tilt angle, and is independent of temperature and axial load. Moreover, since it does not need an additional strain transducer, the proposed tilt sensor is compact in size and has a good linear response with excellent repeatability in a wide dynamic range.

The schematic diagram of the proposed sensor configuration is depicted in Fig.1. It comprises a ceramic ferrule, an FBG written in ECF and a mass. The ECF passes through the ceramic ferrule and keep close contact with it. The center of the ECFBG is just at the bottom of the ceramic ferrule. The upper section of the ECFBG is housed inside the ceramic ferrule, and the lower part of the ECFBG is outside. A small mass is attached to the end of the ECF to increases the bend response under gravity. Since the core is not at the geometrical center of the cross-section of the cladding, the ECFBG is intrinsically sensitive to inclination.

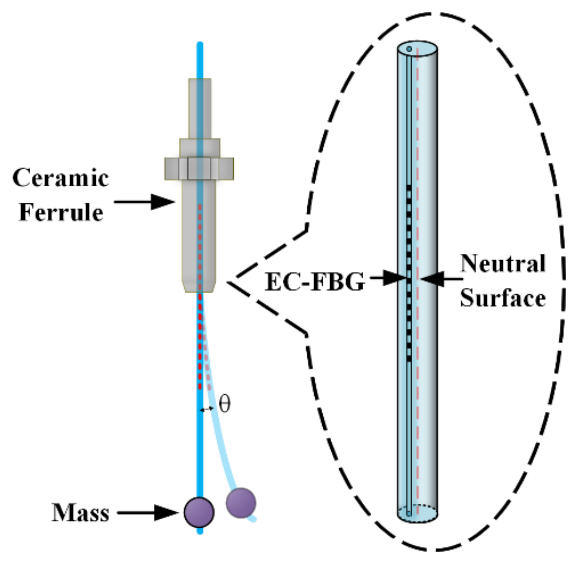

Fig.1. Schematic diagram of the proposed ECFBG tilt sensor.

The working principle of the proposed sensor is based on the strain difference along the length of the ECFBG. When the sensor tilts, the two halves of the ECFBG respond differently because the strains produced by the clamped and unclamped sections are different, and can be viewed as two cascaded sub-ECFBGs with two different resonant peaks. The peak relating to the ECFBG section outside the ceramic ferrule (for convenience denoted as "FBG1") shifts with inclination due to the longitudinal strain variation (bending strain) related to the offset location of the eccentric core. While the peak corresponding to the part of ECFBG placed inside the ceramic ferrule (FBG2) remains unchanged at any tilt angle. This is because the ECF keeps straight, so that there is no tilt-induced longitudinal strain.

A length of ECF was clamped by the ceramic ferrule at one end, so that the gravitational force acts on the resulting fiber cantilever, which bends the free end of the fiber. Two factors contribute to the bending of the fiber cantilever: loading due to the mass per unit length and loading from the mass at the fiber end. According to the theory of material mechanics and under the hypotheses that stress is proportional to strain, the curvature is small and shear stresses can be neglected, when the sensor tilts to angle $\theta$ with respect to the vertical direction, the magnitude of the bending moment in the section containing the FBG1 can be calculated as

$$
M=\left(\frac{q l_{1}^{2}}{2}+P l_{2}\right) \sin \theta
$$

where $M$ is the magnitude of the bending moment, $l_{1}$ is the distance between the FBG1 and the fiber end, $l_{2}$ is the distance between the mass barycenter and FBG1, $q$ and $P$ are the magnitude of the weight per unit length of the fiber and the magnitude of the weight of the mass, respectively.

Under Kirchhoff's rod hypothesis, which states the cross sections remain plane and normal to the centerline and that the centerline is inextensible [18], the bending axial strain on the eccentric core as well as FBG1 can be obtained as

$$
\varepsilon_{z}=\frac{M d}{E I_{x, y}}
$$

where $\varepsilon_{z}$ is the bending strain in the direction of the fiber axis $z$, $d$ is the perpendicular distance between the eccentric core and the neutral axis, $E$ is the Young's modulus of the fiber and $I_{x, y}$ is the second moment of area of inertia of the cross-section about the directions perpendicular to the fiber axis. The approach followed above (2) can determine the bending strain in a fiber under simple bending without taking into account the contribution of the longitudinal strain due to temperature variations and axial load. Nevertheless, it is perfectly suitable for this case, since the sensor detects the peak split, which is the shift between FBG1 and FBG2, and, thus, the effects of temperature and axial load, which affect both FBGs equally, are automatically compensated. Therefore, the wavelength shift of FBG1 can be expressed as

$$
\frac{\Delta \lambda_{1}}{\lambda_{0}}=\left(1-p_{e}\right) \varepsilon+\frac{\partial \lambda_{1}}{\partial T} \Delta T
$$

where $p_{e}$ is the photoelastic constant of the ECF, which is approximately 0.22 for silica fiber. $\lambda_{0}$ is the original ECFBG Bragg wavelength. As the FBG2 is fixed by the ceramic ferrule, the corresponding wavelength shift is only affected by temperature

$$
\frac{\Delta \lambda_{2}}{\lambda_{0}}=\frac{\partial \lambda_{2}}{\partial T} \Delta T
$$

where $\partial \lambda_{1} / \partial T$ and $\partial \lambda_{2} / \partial T$ are temperature coefficients of the two ECFBG halves, respectively. In theory, since the two halves are close to each other, the temperature coefficients of both can be considered identical, as confirmed by the following experiment results. Therefore, according to (1) to (4), the wavelength separation between two peaks can be given by

$$
\begin{aligned}
\Delta \lambda & =\Delta \lambda_{1}-\Delta \lambda_{2} \\
& =\frac{\lambda_{0} d\left(1-p_{e}\right)}{E I}\left(\frac{q l_{1}^{2}}{2}+l_{2} P\right) \sin \theta
\end{aligned}
$$

It can be seen that $\Delta \lambda$ is proportional to $\sin \theta$, and has an opposite sign at positive and negative inclinations. The magnitude and direction of the inclination can therefore be readily calculated from the wavelength separation between the two peaks. In addition, thanks to the measurement of the wavelength separation of two peaks instead of the absolute wavelength, the crosssensitivity arising from thermo-optic effects in ECFBG is completely eliminated. In fact, thermal effects tend to shift both FBGs (1 and 2) in the same direction by the same amount, thus making the wavelength separation temperature independent.

Since the ECF was not available in the laboratory, in our proofof-concept experiment, the ECF was substituted by a commercial four-core fiber (FCF) (Fibercore Ltd) to produce the proposed tilt 
sensor. The FCF consisted of four single-mode cores arranged in a square pattern with a core separation of $50 \mu \mathrm{m}$ and a cladding diameter of $125 \pm 1 \mu \mathrm{m}$. In order to increase the photosensitivity, the FCF was hydrogen-loaded at ambient temperature for two weeks at 50 bar pressure. Then 20mm-long FBGs with $\sim 40 \%$ reflectivity were written into the four cores simultaneously by a $244 \mathrm{~nm}$ CW frequency-doubled argon-ion laser [19,20]. To manufacture the sensor, the FCF was first positioned through a ceramic ferrule with $125 \mu \mathrm{m}$ inner diameter and fastened by epoxy resin. One half of the ECFBG was inside in ceramic ferrule and the other half outside. A small ball of epoxy resin was fixed just beyond the end of the ECF, forming the proof mass of a fiber cantilever to increase sensitivity. The distance between the bottom of the ceramic ferrule and the barycenter of the epoxy ball is $\sim 5.5$ $\mathrm{cm}$. To eliminate the unwanted background noise caused by reflection from the fiber tip, the end of the FCF was coated with refractive Index Matching Liquid (IML).

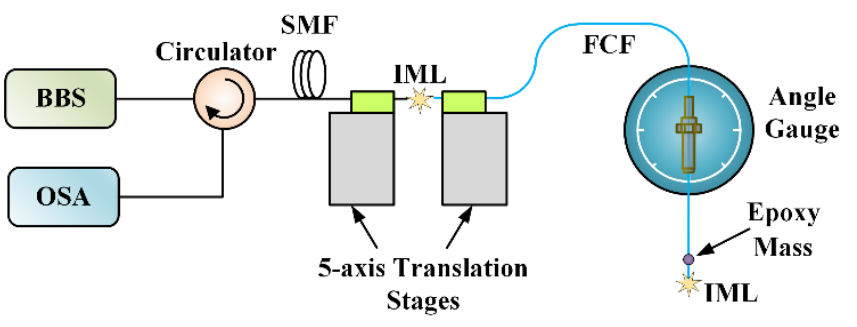

Fig. 2. Experimental setup for sensor characterization

Fig. 2 shows the inclination measurement setup. In order to obtain strain signals from individual cores, a standard single-mode fiber (SMF) and the FCF containing the ECFBG were placed on top of a 5-axis translation stage to carry out core-alignment. The leadin of the SMF and ECF were cleaved and quite close to each other. The IML was filled in between two fibers to increase the coupling efficiency. For practical applications, splicing the SMF to an ECF or the use of a purpose-built fan-out provides easier access to the individual cores [21]. To characterize the tilt sensor response, the sensor was mounted on a standard angle gauge with a resolution of $0.001^{\circ}$. The plane defined by the eccentric core and the geometrical center was parallel to the tilt direction. A broadband light source (BBS) was used to illuminate the tilt sensor, and the reflection spectrum of ECFBG was then monitored by an optical spectrum analyzer (OSA) with a wavelength resolution of $0.01 \mathrm{~nm}$ by means of an optical circulator.

Fig.3 shows the reflection spectra of the ECFBG at three different tilt angles. It can be seen that the Bragg wavelength of the ECFBG splits into two peaks in the reflection spectrum when the tilt angle is not equal to zero. The peak wavelength of $1546.1 \mathrm{~nm}$ is from the fixed part of the ECFBG (FBG2), which remains unchanged during the whole inclination procedure. The other peak is from FBG1, which shifts to a longer (shorter) wavelength with an increasing positive (negative) tilt angle. As the Bragg reflection peak splits into two, the reflectivity is reduced slightly, because the effective length of each sub-ECFBG is reduced. The wavelength separation of the two peaks is plotted against the tilt angle in Fig.4. The tilt angle varied from $-45^{\circ}$ to $+45^{\circ}$ in increments of $5^{\circ}$. It is clear that the wavelength separation has a linear response to tilt angle over a dynamic range between $-45^{\circ}$ and $+45^{\circ}$. The measured sensitivities for the positive and negative inclinations were found

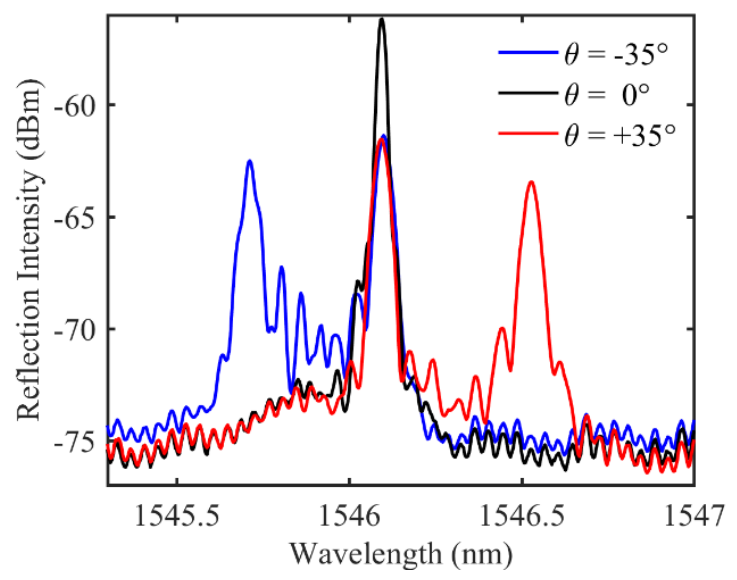

Fig. 3. Spectral responses of ECFBG at three tilt angles

to be $0.012 \mathrm{~nm} /{ }^{\circ}$ and $0.011 \mathrm{~nm} /{ }^{\circ}$, respectively. The theoretical sensitivity of the tilt sensor is $0.0143 \mathrm{~nm} /{ }^{\circ}$, derived from (5), which is very close to the experimental results. The discrepancy between the theoretical predictions and experimental measurements is considered reasonable mainly given by experimental uncertainties. Limited by the interrogator's wavelength resolution of $0.01 \mathrm{~nm}$, the highest resolution of the inclinometer is $\sim 0.83^{\circ}$. In fact, the sensitivity of the tilt sensor can be adjusted by several parameters, such as the distance between the eccentric core and the neutral axis, the length of the fiber cantilever and the weight of the epoxy ball. Accordingly, the accuracy of tilt sensor can be further improved by higher sensitivity as well as by adopting an interrogator with a higher wavelength resolution.
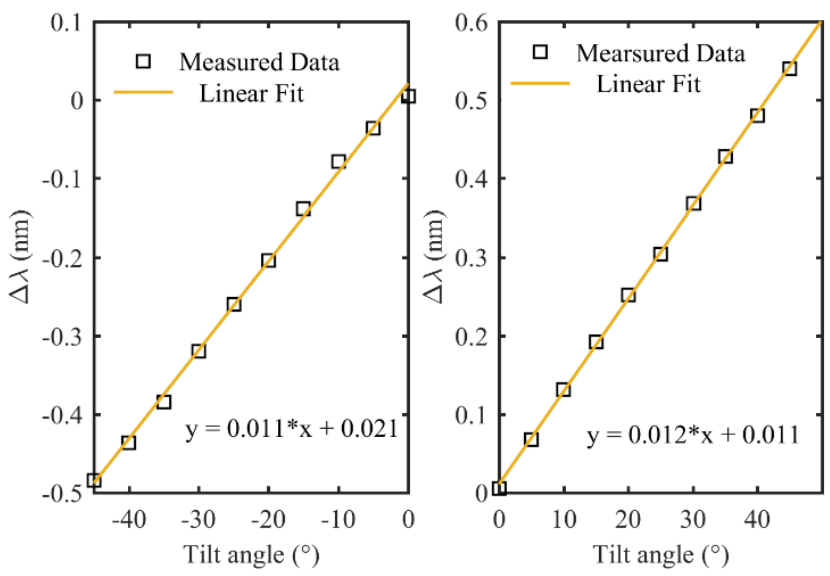

Fig. 4. Measured wavelength separation of EC-FBG versus tilt angle.

To examine the influence of temperature change on sensor performance, Figure 5 shows the variations of $\Delta \lambda$ against temperature at two different tilt angles. The tilt sensor was placed inside a temperature-controlled container in which the temperature varied from $27^{\circ} \mathrm{C}$ to $65^{\circ} \mathrm{C}$. Each temperature value was maintained for 60 seconds to guarantee both parts of the ECFBG were subject to the same temperature. The results show that the wavelength separations obtained at different temperature values are quite stable. The maximum discrepancy of the 
wavelength shift was found to be less than $\pm 0.002 \mathrm{~nm}$, which means that the tilt sensor has a very low temperature crosssensitivity.

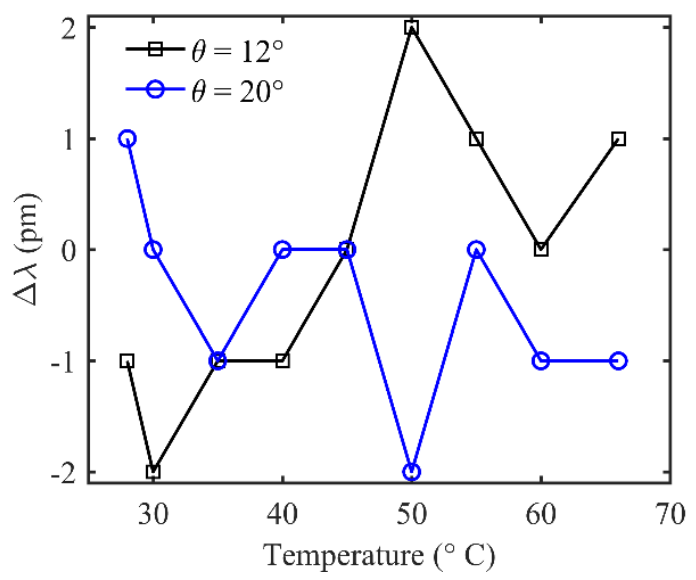

Fig. 5. Thermal response of the tilt sensor depicting the change in wavelength separation when it is fixed at two different tilt angles.

In order to investigate the sensor's repeatability and reliability, an angle cycling test was conducted. The tilt angle was initially set at $-45^{\circ}$, then went forward to $+45^{\circ}$, and finally returned backward to $-45^{\circ}$, with a step size of $5^{\circ}$. The test was repeated with 3 cycles, as shown in Fig.6. The maximum discrepancy is observed to be less than $\pm 0.005 \mathrm{~nm}$, with the corresponding angular uncertainty of $\pm 0.41^{\circ}$ for both increasing and decreasing inclinations, showing that the proposed sensor is highly reversible with very low hysteresis.

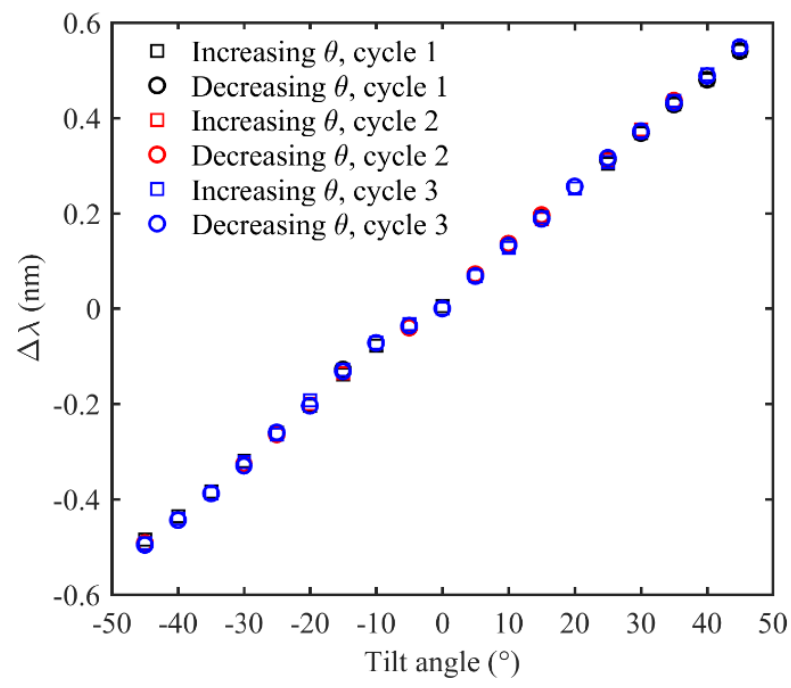

Fig.6. Repeated testing of wavelength separation versus tilt angle.

In summary, an original, simple and compact tilt sensor based on a single ECFBG with the ability to determine the 1-D tilt angle and direction is reported in this paper. The proposed sensor does not use an additional pendulum or cantilever for strain transduction, which greatly reduces strain-transfer-induced measurement errors. The experimental results show that the proposed device has a good linear response in the wide dynamic range of $\pm 45^{\circ}$ with a sensitivity of $0.012 \mathrm{~nm} /{ }^{\circ}$ and $0.011 \mathrm{~nm} /{ }^{\circ}$ for positive and negative inclinations, respectively. Good repeatability and reliability was obtained, with a maximum angular uncertainty of $\pm 0.41^{\circ}$, which indicates a low degree of hysteresis. It should be pointed out that the sensitivity of the proposed tilt sensor is lower compared with the previous works based on common FBG $[10,11]$. However, the proposed device has more compact structure, larger dynamic range and simpler interrogation operation. The minimum angle that the tilt sensor can detect in our current experiment is $\pm 4^{\circ}$, the tilt sensor with better discrimination capability at small tilt angle can be achieved by increasing the distance between the eccentric core and the neutral axis, the length of the fiber cantilever and the weight of the epoxy ball. Furthermore, the proposed tilt sensor presents a temperature-insensitive characteristic, which means it can be used in thermally uncontrolled environments. For practical applications, the sensor can be enclosed in a viscous fluid-filled cylinder to dampen environmental vibrations.

Funding. This work was supported in part by the National Natural Science Foundation of China (61405166, 61775185), Sichuan Science and Technology Program (2018HH0002) and 111 Plan (B18045), and the Spanish Ministry of Economy and Competitiveness under the project DIMENSION TEC2017 88029$\mathrm{R}$, and the ITN-FINESSE framework, funded by the European Union's Horizon 2020 Research and Innovation Program under the Marie Sklodowska-Curie Action Grant Agreement N 722509.

\section{References}

1. M. R. Islam, M. Bagherifaez, M. M. Ali, H. K. Chai, K.-S. Lim, and H. Ahmad, IEEE Trans. Instrum. Meas. 64, 3510 (2015).

2. Y. Guo, D. Zhang, J. Fu, S. Liu, S. Zhang, and F. Zhu, Sensor Rev. 35, 348 (2015).

3. C. Lin and S. Kuo, Sens. Actuators A, Phys. 143, 113 (2008).

4. P. C. Hu, J. H. Guo, J. B. Tan, IEEE Trans. Ind. Electron. 63, 2469 (2016).

5. B. Ando, A. Ascia, S. Baglio, IEEE Transactions on Instrumentation and Measurement 59, 558 (2010).

6. G. A. Miller, IEEE Photon. Technol. Lett. 27, 1289 (2015).

7. Y. Yang, E. Wang, K. Chen, Z. Yu and Q. Yu, IEEE Sensors J. 19, 2162 (2019).

8. Q. Zhang, T. Zhu, F. Yin, and K. S. Chiang, IEEE Photon. Technol. Lett. 26, 1049 (2014).

9. Y. Zhuang, Y. Chen, C. Zhu, R. E. Gerald, and J. Huang, Opt. Express. 26, 2546 (2018).

10. B. O. Guan, H. Y. Tam, and S. Y. Liu, IEEE Photon. Technol. Lett. 16, 224 (2004).

11. H. Bao, X. Dong, C. Zhao, L. Shao, C. C. Chan, and P. Shun, Opt. Commun. 283, 968 (2010).

12. X. Dong, C. Zhan, K. Hu, P. Shum, and C. C. Chan, IEEE Photon. Technol. Lett. 17, 2394 (2005).

13. R. Yang, H. Bao, S. Zhang, K. Ni, Y. Zheng, and X. Dong, IEEE Sensors J. 15, 6381 (2015).

14. H. J. Chen, L. Wang, and W. F. Liu, Appl. Opt. 47, 556 (2008).

15. H. Y. Au, S. K. Khijwania, H. Y. Fu, W. H. Chung, and H. Y. Tam, J. Lightwave Technol. 29, 1714 (2011).

16. P. Munendhar, R. Aneesh, and S. K. Khijwania, Appl. Opt. 53, 3574 (2014).

17. W. N. MacPherson, G. M. H. Flockhart, R. R. J. Maier, J. S. Barton, J. D. C. Jones, D. Zhao, L. Zhang and I. Bennion, Meas. Sci. Technol. 15, 1642 (2004).

18. O. M. O'Reilly, Modeling Nonlinear Problems in the Mechanics of Strings and Rods, Springer, (2017) 
19. D. Zheng, J. Madrigal, H. Chen, D. Barrera, and S. Sales, Opt. Lett. 42, 3710 (2017).

20. D. Zheng, J. Madrigal, D. Barrera, S. Sales, and J. Capmany, IEEE Photon. Technol. Lett. 29, 1707 (2017).

21. J. Kong, X. Ouyang, A. Zhou, H. Yu, and L. Yuan, J. Lightwave Technol. 34, 3288 (2016). 
1. M. R. Islam, M. Bagherifaez, M. M. Ali, H. K. Chai, K.-S. Lim, and H. Ahmad, "Tilted fiber Bragg grating sensors for reinforcement corrosion measurement in marine concrete structure," IEEE Transactions on Instrumentation and Measurement, 64(12), 3510-3516, 2015.

2. Y. Guo, D. Zhang, J. Fu, S. Liu, S. Zhang, and F. Zhu, "Development and operation of a fiber Bragg grating based Online monitoring strategy for slope deformation," Sensor Review, 35(4), 348-356, 2015.

3. C. Lin and S. Kuo, "Micro-impedance inclinometer with wide-angle measuring capability and no damping effect," Sensors and Actuators APhysical, 143(1), 113-119, 2008.

4. P. C. Hu, J. H. Guo, and J. B. Tan, "An annular planar-capacitive tilt sensor with a $360^{\circ}$ measurement range," IEEE Transactions on Industrial Electronics, 63(4), 2469-2476, 2016.

5. B. Ando, A. Ascia, S. Baglio, "A ferrofluidic inclinometer in the resonant configuration," IEEE Transactions on Instrumentation and Measurement 59(3), 558-564, 2010.

6. G. A. Miller, "Fabrication of a multifiber optical inclinometer," IEEE Photonics Technology Letters, 27(12), 1289-1292, 2015.

7. Y. Yang, E. Wang, K. Chen, Z. Yu and Q. Yu, "Fiber-optic Fabry-Perot sensor for simultaneous measurement of tilt angle and vibration acceleration," IEEE Sensor Journal, 19(6), 2162-2169, 2019

8. Q. Zhang, T. Zhu, F. Yin, and K. S. Chiang, "Temperature-insensitive realtime inclinometer based on an etched fiber Bragg grating," IEEE Photonics Technology Letters, 26(10), 1049-1052, 2014.

9. Y. Zhuang, Y. Chen, C. Zhu, R. E. Gerald, and J. Huang, "Probing changes in tilt angle with 20 nanoradian resolution using an extrinsic Fabry-Pérot interferometer-based optical fiber inclinometer," Optics Express, 26(6), 2546-2556, 2018.

10. B. O. Guan, H. Y. Tam, and S. Y. Liu, "Temperature independent fiber Bragg grating tilt sensor," IEEE Photonics Technology Letters, 16(1), 224226, 2004

11. H. Bao, X. Dong, C. Zhao, L. Shao, C. C. Chan, and P. Shun, "Temperatureinsensitive FBG tilt sensor with a large dynamic range," Optics Communications, 283(6), 968-970, 2010.

12. X. Dong, C. Zhan, K. Hu, P. Shum, and C. C. Chan, "Temperatureinsensitive tilt sensor with strain-chirped fiber Bragg gratings," IEEE Photonics Technology Letters. 17(11), 2394-2396, 2005.

13. R. Yang, H. Bao, S. Zhang, K. Ni, Y. Zheng, and X. Dong, "Simultaneous measurement of tilt angle and temperature with pendulum based fiber Bragg grating sensor," IEEE Sensors Journal, 15(11), 6381-6384, 2015.

14. H. J. Chen, L. Wang, and W. F. Liu, "Temperature-insensitive fiber Bragg grating tilt sensor," Applied Optics, 47(4), 556-560, 2008.

15. H. Y. Au, S. K. Khijwania, H. Y. Fu, W. H. Chung, and H. Y. Tam, "Temperature-insensitive fiber Bragg gratings based tilt sensor with large dynamic range," Journal of Lightwave Technology. 29(11), 1714-1720, 2011.

16. P. Munendhar, R. Aneesh, and S. K. Khijwania, "Development of an alloptical temperature insensitive nonpendulum-type tilt sensor employing fiber Bragg gratings," Applied Optics. 53(16), 3574-3580, 2014.

17. W. N. MacPherson, G. M. H. Flockhart, R. R. J. Maier, J. S. Barton, J. D. C. Jones, D. Zhao, L. Zhang and I. Bennion, "Pitch and roll sensing using fibre Bragg gratings in multicore fibre," Measurement Science and Technology, 15, 1642-1646, 2004.

18. O. M. O'Reilly, Modeling Nonlinear Problems in the Mechanics of Strings and Rods, Springer, (2017)

19. D. Zheng, J. Madrigal, H. Chen, D. Barrera, and S. Sales, "Multicore fiber Bragg grating based directional curvature sensor interrogated by broadband source with sinusoidal spectrum," Optics Letters, 42(18), 3710-3713, 2017.

20. D. Zheng, J. Madrigal, D. Barrera, S. Sales, and J. Capmany, "Microwave Photonic Filtering for Interrogating FBG-based Multicore Fiber Curvature Sensor," IEEE Photonics Technology Letters, 29(20), 1707-1710, 2017.

21. J. Kong, X. Ouyang, A. Zhou, H. Yu, and L. Yuan, "Pure directional bending measurement with a fiber Bragg grating at the connection joint of eccentric-core and single-mode fibers," Journal of Lightwave Technology, 34(14), 3288 - 3292, 2016. 